\title{
TOPOLOGICAL TYPES OF PFAFFIAN MANIFOLDS
}

\author{
MASATO FUJITA AND MASAHIRO SHIOTA
}

\begin{abstract}
Let $\Omega=\left(\omega_{1}, \ldots, \omega_{n-k}\right)$ be differential 1-forms with polynomial coefficients in $\mathbf{R}^{n}$. A Pfaffian manifold of $\Omega$ is by definition a maximal integral $k$-manifold of $\Omega$. It is shown that the number of homeomorphism classes of all Pfaffian manifolds of Rolle Type of $\Omega$ is finite and, moreover, bounded by a computable function in variables $n, k$ and the degree of $\omega_{1}, \ldots, \omega_{n-k}$. Finiteness is proved also in any o-minimal structure.

We give also an example of a semi-algebraic $C^{1}$ differential form on a semialgebraic $C^{2} 3$-manifold whose Pfaffian manifolds have homeomorphism classes of the cardinality of continuum. Hence the cardinality of all manifolds is the continuum (not countable).
\end{abstract}

\section{$\S 1$. Introduction}

This paper treats of Pfaffian manifolds in the o-minimal setting. See [15], [16] for the theory of o-minimal structure. We have to give definitions and notations before we introduce and discuss our results.

Let $M$ be a $C^{2}$ manifold. Let $\Omega=\left\{\omega_{1}, \ldots, \omega_{q}\right\}$ be $C^{1}$ differential 1forms on $M$. A Pfaffian manifold of $\Omega$ is a maximal connected $C^{1}$ manifold $V$ immersed in $M$ such that $T_{x} V=\bigcap_{i=1}^{q} \operatorname{ker}\left(\omega_{i}\right)$ for all $x \in V$. Remark that it is not necessarily a submanifold of $M$. In particular, if $q=1$, we call it a Pfaffian hypersurface. We call $(V, \Omega, M)$ a Pfaffian manifold for short. A foliation defined by $\Omega$ is a foliation whose leaves are Pfaffian manifolds of $\Omega$.

A Pfaffian manifold $(V, \Omega, M)$ is of Rolle type if the following Rolle Property is satisfied.

- Let $\gamma:[0,1] \rightarrow M$ be a $C^{1}$ curve such that $\gamma(0)$ and $\gamma(1)$ are contained in $V$. Then, for any $i=1, \ldots, q$, there exists $t_{i} \in[0,1]$ such that $\omega_{i}\left(\gamma\left(t_{i}\right)\right) \cdot \gamma^{\prime}\left(t_{i}\right)=0$.

Received October 25, 2001.

Revised November 18, 2002.

2000 Mathematics Subject Classification: Primary 03C64, 14P10; Secondary 58A17. 
It is easy to see that a Pfaffian manifold of Rolle type is a submanifold of $M$. This notion of Pfaffian manifold of Rolle type is a generalization of the notion of Rolle hypersurface due to Khovanskii and Mossu-Roche [6], [10], [11]. Many remarkable studies for Rolle hypersurface are published, for example, [2], [3], [6], [7], [8], [9], [10], [11], [14], [17]. A Pfaffian manifold of non-Rolle type is a Pfaffian manifold which is not of Rolle type. For some $(M, \Omega)$, almost all Pfaffian manifolds are of Rolle type. For example, any Pfaffian manifold is of Rolle type if $M$ is simply connected, if $\Omega=\{\omega\}$, i.e. $q=1$, and if $\omega$ is nonsingular and integrable everywhere (Haefliger [5]). We call a Pfaffian manifold of Rolle type a Rolle leaf when $\Omega$ defines a foliation, that is, $\Omega$ is integrable everywhere on $M$.

Consider an o-minimal expansion $\tilde{\mathbb{R}}$ of the real field. A definable $C^{r}$ manifold $M$ is a subset of some $\mathbb{R}^{n}$ which is both definable and a $C^{r}$ submanifold of $\mathbb{R}^{n}$. Consider a differential 1-form on $M$. Identify it with the image of its dual under the natural inclusion $T_{*} M \hookrightarrow T_{*} \mathbb{R}^{n}$ with the usual inner product. A differential 1-form on $M$ is definable if its coefficients are definable.

Now we introduce and discuss our results. We show in Section 2 that a Pfaffian manifold of Rolle type is a connected component of an intersection of Pfaffian hypersurfaces of Rolle type.

Speissegger showed in his paper [14] that there exists an o-minimal expansion $\mathcal{P}(\tilde{\mathbb{R}})$ of $\tilde{\mathbb{R}}$ such that $V$ is definable in it for any Pfaffian hypersurface $\left(V, \Omega=\left\{\omega_{1}\right\}, M\right)$ (namely, in the case when $q=1$ ) of Rolle type if $\omega_{1}$ and $M$ are definable in it. Since a Pfaffian manifold of Rolle type is a connected component of an intersection of Pfaffian hypersurfaces of Rolle type, we have the following corollary.

Corollary 1.1. Let $\tilde{\mathbb{R}}$ be an o-minimal structure on $\mathbb{R}$. Then, for a $\mathcal{P}(\tilde{\mathbb{R}})$-definable $C^{2}$ manifold and for a family $\Omega$ of $\mathcal{P}(\tilde{\mathbb{R}})$-definable differential 1-forms on it, any Pfaffian manifold of $\Omega$ of Rolle type is also $\mathcal{P}(\tilde{\mathbb{R}})$-definable.

The o-minimal expansion with the above property is called the Pfaffian closure of $\tilde{\mathbb{R}}$.

Corollary 1.1 and its proof have a good application. Re-scrutinizing its proof closely, we can prove the following theorems concerning the topological types of Pfaffian manifolds of Rolle type. 
THEOREM 1.2. Let $\tilde{\mathbb{R}}$ be an o-minimal structure on $\mathbb{R}$. Let $M$ be a $\mathcal{P}(\tilde{\mathbb{R}})$-definable $C^{2}$ manifold and $\Omega$ be a finite family of $\mathcal{P}(\tilde{\mathbb{R}})$-definable $C^{1}$ differential 1-forms on it. Then any Pfaffian manifold of $\Omega$ of Rolle type is homeomorphic to some nonsingular affine real algebraic set and the homeomorphism can be chosen to be definable in $\mathcal{P}(\tilde{\mathbb{R}})$.

THEOREM 1.3. Let $\tilde{\mathbb{R}}, M$ and $\Omega$ be the same as above. Let $\mathcal{T}_{\Omega}$ be the set of all Pfaffian manifolds of $\Omega$ of Rolle type. Then the quotient set of $\mathcal{T}_{\Omega}$ up to $\mathcal{P}(\tilde{\mathbb{R}})$-definable homeomorphism is finite.

In particular,

THEOREM 1.4. Let $M$ be a compact real analytic manifold. Let $\Omega$ be a finite family of analytic differential 1-forms on $M$. Let $\mathcal{T}_{\Omega}^{\text {ana }}$ be the set of all Pfaffian manifolds of $\Omega$ of Rolle type. Then any Pfaffian manifold of $\Omega$ of Rolle type is homeomorphic to some affine nonsingular real algebraic set and the quotient set of $\mathcal{T}_{\Omega}^{\text {ana }}$ up to homeomorphism is finite.

The algebraic case is more simple.

THEOREM 1.5. Let $n, d, q$ be positive integers. Consider all the differential 1-forms on $\mathbb{R}^{n}$ with polynomial coefficients of total degree $\leq d$. Let $\mathcal{T}_{n, d, q}$ denote the set of all Pfaffian manifold of such $q 1$-forms of Rolle type. Then any element of $\mathcal{T}_{n, d, q}$ is homeomorphic to a nonsingular real algebraic set, the quotient set of $\mathcal{T}_{n, d, q}$ up to homeomorphism is finite and the number of elements of this quotient set is bounded by some computable function $l(n, d, q)$.

The latter claim of this theorem is the direct corollary of [2, Théorème 1] in the case when $q=1$.

Pfaffian manifolds of Rolle type have some good properties as it is described above. What about the non-Rolle case? The research of Pfaffian manifolds of non-Rolle type can be reduced to the Rolle case in the following sense.

Proposition 1.6. Let $\tilde{\mathbb{R}}$ and $M$ be the same as above. Let $\omega$ be a nonsingular integrable $\mathcal{P}(\tilde{\mathbb{R}})$-definable $C^{1}$ differential 1 -form on $M$. Then there exists a finite definable open covering $\left\{U_{i}\right\}$ of $M$ such that any Pfaffian manifold of $\left.\omega\right|_{U_{i}}$ is of Rolle type. 
Proposition 1.7. Let $M$ be a semialgebraic $C^{2}$ manifold, and let $\omega$ be a nonsingular and integrable semialgebraic $C^{1}$ differential 1-form on $M$. Then there exists a finite semialgebraic open covering $\left\{U_{i}\right\}$ of $M$ such that any Pfaffian manifold of the restriction $\left.\omega\right|_{U_{i}}$ is of Rolle type.

Proposition 1.8. Let $M$ be a compact real analytic manifold, and let $\omega$ be a nonsingular and integrable analytic differential 1-form on $M$. Then there exists a finite open covering $\left\{U_{i}\right\}$ of $M$ with the same property in the above theorem.

On the mere face of these theorems, the topological types of leaves of a foliation defined by a semialgebraic or an analytic differential 1-form seem to be finite or at most countable. Nevertheless, a foliation defined by a differential 1-form may have more topological types. Incidentally, it was not known whether the topological types of all 2-dimensional manifolds are countable or not.

THEOREM 1.9. There exist a semialgebraic $C^{2}$ manifold of dimension 3 and a semialgebraic $C^{1}$ differential 1-form on it such that the quotient set of the set of all Pfaffian manifolds of the form up to homeomorphism has cardinality of the continuum.

THEOREM 1.10. There exist a compact real analytic manifold of dimension 3 and an analytic differential 1-form on it with the following property. The quotient set of the set of Pfaffian manifolds of the form up to homeomorphism has cardinality of the continuum.

Remark that the differential 1-form given in the last theorem has the nonempty singular locus. We do not know an example without singularities.

We are grateful to the referee for the valuable advice.

\section{§2. Generalized Khovanskii's theory and Pfaffian closure}

We first show the following proposition which is proposed by the referee. Remark that Corollary 1.1 is the direct corollary of it.

Proposition 2.1. A Pfaffian manifold of Rolle type is a connected component of an intersection of Pfaffian hypersurfaces of Rolle type. 
Proof. Let $M$ be a $C^{2}$ manifold and $\Omega=\left\{\omega_{1}, \ldots, \omega_{q}\right\}$ be a family of $C^{1}$ differential 1-forms. Fix an arbitrary Pfaffian manifold $(V, \Omega, M)$ of Rolle type. We may assume without loss of generality that $V$ is contained in $M \backslash \bigcup_{i=1}^{q} \operatorname{Sing}\left(\omega_{i}\right)$, where $\operatorname{Sing}\left(\omega_{i}\right)$ denotes the singular locus of $\omega_{i}$. We have only to show that each $\omega_{i}$ is integrable on a neighborhood of $V$, namely

$$
V \subset M \cap\left(\bigcap_{i=1}^{q}\left\{\omega_{i} \wedge d \omega_{i}=0\right\}\right) .
$$

Let $P \in M$ such that $\omega_{i} \wedge d \omega_{i}(P) \neq 0$. Since $\omega_{i}$ is nonsingular at $P$, there exists a local $C^{1}$ coordinates $\left(x_{1}, \ldots, x_{n}\right)$ around $P \in M$ such that $P$ is the origin and $\omega_{i}=d x_{1}+x_{2} d x_{3}+\sum_{i=4}^{n} a_{i} d x_{i}$ for some $C^{1}$ functions $a_{4}, \ldots, a_{n}$ by Darboux's Theorem.

Consider the closed curve $\gamma_{\varepsilon}:[0,2 \pi] \rightarrow \mathbb{R}^{n}$ defined by

$$
\gamma_{\varepsilon}(t)=\left(\varepsilon^{2} \sin (2 t) / 2,2 \varepsilon \sin (t), \varepsilon(1-\cos (t)), 0, \ldots, 0\right),
$$

where $\varepsilon$ is a sufficiently small positive number. Then the curve verify:

$$
\omega_{i}\left(\gamma_{\varepsilon}(t)\right) \cdot \gamma_{\varepsilon}^{\prime}(t)=\varepsilon^{2} \text { and } P=\gamma_{\varepsilon}(0)
$$

Namely, $P \notin V$.

Theorem 1.2 and the following corollaries are the consequence of Proposition 2.1.

Proof of Theorem 1.2. Immediate by [13, Theorem II.5.11], [12, Remark VI.2.11] and Corollary 1.1.

Corollary 2.2. Consider an o-minimal structure on $\mathbb{R}$. Let $\Omega_{i}=$ $\left\{\omega_{i 1}, \ldots, \omega_{i q(i)}\right\}$ be definable $C^{1}$ differential 1 -forms on a definable $C^{2}$ manifold $M$ for $i=1, \ldots, p$. Let $A$ be a definable subset of $M$. Then there exists an integer $N$ determined by $M, \Omega_{i}, i=1, \ldots, p$, and $A$ such that, for any Pfaffian manifolds $V_{i}$ of $\Omega_{i}$ of Rolle type, the number of connected components of $A \cap V_{1} \cap \cdots \cap V_{p}$ is smaller than $N$.

Proof. Obvious by Proposition 2.1 and [14, Theorem 2.6].

Consider an o-minimal structure on $\mathbb{R}$. Definable sets $X_{\lambda} \subset \mathbb{R}^{n}$ are with parameter $\lambda \in \mathbb{R}^{m}$ if $\bigcup_{\lambda \in \mathbb{R}^{m}} X_{\lambda} \times\{\lambda\}$ is a definable subset of $\mathbb{R}^{n} \times \mathbb{R}^{m}$. We say that a definable set $M_{\lambda}$ with parameter is a definable $C^{2}$ manifold with parameter if $\bigcup_{\lambda} M_{\lambda}$ and $M_{\lambda}$ are $C^{2}$ manifolds for all $\lambda$. Similarly, definable $C^{1}$ differential 1-forms on a definable $C^{2}$ manifold with parameter are defined to be with parameter. 
Corollary 2.3. Consider an o-minimal structure on $\mathbb{R}$. Let $X_{\lambda}, M_{\lambda}$ and $\Omega_{i, \lambda}=\left\{\omega_{i 1, \lambda}, \ldots, \omega_{i q(i), \lambda}\right\}, i=1, \ldots, p$, be definable subsets of $\mathbb{R}^{n}$ with parameter $\lambda \in \mathbb{R}^{m}$, definable $C^{2}$ submanifold of $\mathbb{R}^{n}$ with parameter and definable $C^{1}$ differential 1-forms with parameter on $M_{\lambda}$, respectively. Then there exists an integer $N$ determined only by $M, X$ and $\Omega_{i, \lambda}$ such that the number of connected components of $X_{\lambda} \cap V_{1} \cap \cdots \cap V_{p}$ is smaller than $N$ for any $\lambda$ and for any Pfaffian manifolds $V_{i}$ of $\Omega_{i, \lambda}$ of Rolle type.

Proof. We show this corollary in the same way as [11, Théorème $\left.1_{\lambda}\right]$, using Proposition 2.1 and [14, Theorem 2.6].

Set $M=\bigcup_{\lambda} M_{\lambda}$. Apply the cylindrical definable $C^{2}$ cell decomposition to $M$. Then $M$ is a finite number of definable $C^{2}$ manifolds $M_{j}$ such that $M_{j},\left\{\lambda ; M_{j, \lambda} \neq \emptyset\right\}$ and each $M_{j, \lambda}$ are definably $C^{2}$ diffeomorphic to Euclidean spaces. Hence we may assume that $M=\mathbb{R}^{m} \times \mathbb{R}^{s}$ and $\mathbb{R}^{s}$ is the parameter space.

We may view the Pfaffian manifold $V_{i}$ of $\Omega_{i, \lambda}$ as the Pfaffian manifold of $\Omega_{i}^{\prime}=\Omega_{i, \lambda} \cup\left\{d \lambda_{1}, \ldots, d \lambda_{s}\right\}$. Then each $V_{i}$ is a Pfaffian manifold of $\Omega_{i}^{\prime}$ of Rolle type. Therefore this corollary follows from Corollary 2.2.

Corollary 2.4. For any positive integers $q, n, d$, there exists an integer $N$ satisfying the following property.

- Let $\Omega=\left\{\omega_{i}=\sum_{j=1}^{n} a_{i j} d x_{j}\right\}_{1 \leq i \leq q}$ be semialgebraic $C^{1}$ differential 1 -forms on $\mathbb{R}^{n}$ such that, for any $i, j$, the graph of $a_{i j}$ is of the form

$$
B_{i, j}=\bigcup_{s=1}^{d} \bigcap_{t=1}^{d}\left\{f_{s, t} *_{s, t} 0\right\},
$$

where $f_{s, t}$ are polynomials in $n+1$ indeterminates of total degree $\leq d$ and $*_{s, t} \in\{<,=\}$.

Let $A$ is a semi-algebraic subset of $\mathbb{R}^{n}$ of the form

$$
\bigcup_{s=1}^{d} \bigcap_{t=1}^{d}\left\{g_{s, t} *_{s, t} 0\right\},
$$

where $g_{s, t}$ are polynomials in $n$ indeterminates of total degree $\leq d$ and $*_{s, t} \in\{<,=\}$.

Let $V$ be a u-Pfaffian manifold of $\Omega$ of Rolle type. Then the number of connected components of $A \cap V$ is smaller than $N$. 
Proof. Set $K:=q d^{2}\left(\begin{array}{c}n+1+d \\ d\end{array}\right)+d^{2}\left(\begin{array}{c}n+d \\ d\end{array}\right)$. We identify $\mathbb{R}^{K}$ with the coefficient space of $d^{2}(q+1)$ polynomials. Because there are only finite ways of choice of signs $*_{s, t}$, we have only to show this corollary for fixed signs. Set

$U^{\prime}:=\left\{x \in \mathbb{R}^{K}:\right.$ the set $B_{i, j}$ determined by $x$ is the graph of a $C^{2}$ function on $\mathbb{R}^{n}$ for all $\left.i, j\right\}$.

Then $U^{\prime}$ is semialgebraic. Applying Corollary 2.3, we obtain the corollary.

\section{§3. Sketch of the proof of Theorem 1.3}

One can prove Theorem 1.3 repeating the argument of [14], [15], [16] with an additional consideration described below. Therefore, we only give a sketch of the proof of Theorem 1.3.

Definition 3.1. Let $\Pi_{\iota}$ denote the map from $\mathbb{R}^{n}$ onto $\mathbb{R}^{k}$ given by $\Pi_{\iota}\left(x_{1}, \ldots, x_{n}\right)=\left(x_{\iota(1)}, \ldots, x_{\iota(k)}\right)$ for a strictly increasing map $\iota:\{1, \ldots, k\}$ $\rightarrow\{1, \ldots, n\}$. A submanifold $L$ of $\mathbb{R}^{n}$ is in standard position if there is an integer $d$ such that $\left.\Pi_{\iota}\right|_{L}$ has constant Jacobian rank $d$ for all $\iota$.

Definition 3.2. A subset $W$ of $\mathbb{R}^{n}$ is a basic $\tilde{\mathbb{R}}$-Pfaffian set if $W=$ $A \cap V_{1} \cap \cdots \cap V_{q}$ for families $\Omega_{1}, \ldots, \Omega_{q}$ of finite definable differential 1forms on $\mathbb{R}^{n}$ whose ranks are full, for Pfaffian manifolds $V_{i}$ of $\Omega_{i}$ of Rolle type and for a definable set $A$. This notion is a generalization of [14, Definition 2.10]. We call then $W$ defined by $\Omega_{1}, \ldots, \Omega_{q}$ and $A$. An $\tilde{\mathbb{R}}$ Pfaffian set is a finite union of basic $\tilde{\mathbb{R}}$-Pfaffian sets. Recall the definition of a $\Lambda^{\infty}$-set $([14$, Definition 3.1$])$. We define a $\Lambda_{\mathcal{D}}^{\infty}$-set to be a $\Lambda^{\infty}$-set obtained from Pfaffian sets.

Definition 3.3. Two basic Pfaffian sets are of the same form if they are defined by the same families and the same definable set. Two Pfaffian sets $W$ and $W^{\prime}$ are of the same form if there exist tuples of basic Pfaffian sets $\left(W_{1}, \ldots, W_{l}\right)$ and $\left(W_{1}^{\prime}, \ldots, W_{l}^{\prime}\right)$ with $W=\bigcup_{j=1}^{l} W_{j}$ and $W^{\prime}=\bigcup_{j=1}^{l} W_{j}^{\prime}$ such that $W_{j}$ and $W_{j}^{\prime}$ are of the same form for all $j$. Two $\Lambda_{\mathcal{D}}^{\infty}$-sets, which are obtained from Pfaffian sets $\left(W_{1}, \ldots, W_{l}\right)$ and $\left(W_{1}^{\prime}, \ldots, W_{l}^{\prime}\right)$, respectively, are of the same form if $W_{j}$ and $W_{j}^{\prime}$ are of the same form for all $j$. 
It is obvious the collection of all Pfaffian sets satisfies the axioms of $\Lambda$-sets. Furthermore, the following lemma holds true.

Lemma 3.4. Let $A, B, A^{\prime}$ and $B^{\prime}$ be Pfaffian sets such that $A$ and $A^{\prime}$ (resp. $B$ and $B^{\prime}$ ) are of the same form. Assume that $A$ and $A^{\prime}$ are subsets of $\mathbb{R}^{n}$.

1. $A \cap B$ and $A^{\prime} \cap B^{\prime}$ are of the same form.

2. $A \times B$ and $A^{\prime} \times B^{\prime}$ are of the same form.

3. Let $\pi$ be a permutation of $\{1,2, \ldots, n\}$. Then $\pi(A)$ and $\pi\left(A^{\prime}\right)$ are of the same form.

4. There exist a projection $\Pi: \mathbb{R}^{m} \rightarrow \mathbb{R}^{n}$ and closed Pfaffian subsets $C$, $C^{\prime}$ of $\mathbb{R}^{m}$ of the same form such that $A=\Pi(C)$ and $A^{\prime}=\Pi\left(C^{\prime}\right)$.

5. Let $k$ be a positive integer. There exists an integer $N$ such that the number of connected components of each fibre $\tilde{A}_{x}=\left\{y \in \mathbb{R}^{n-k}\right.$ : $(x, y) \in \tilde{A}\}$ is smaller than $N$ for any $x \in \mathbb{R}^{k}$ and for any Pfaffian set $\tilde{A}$ which together with $A$ are of the same form.

6. There exist $C^{1}$ manifolds $B_{1}, \ldots, B_{l}, B_{1}^{\prime}, \ldots, B_{l}^{\prime}$ in standard position with $A=\bigcup_{j=1}^{l} B_{j}$ and $A^{\prime}=\bigcup_{j=1}^{l} B_{j}^{\prime}$ such that $B_{j}$ and $B_{j}^{\prime}$ are Pfaffian sets of the same form for all $j$.

Proof. Claims 1-4 are clear. Claim 5 is nothing but Corollary 2.2. Claim 6 is a direct consequence of [14, Corollary 2.9].

The proof of that intersection, union and difference of two $\Lambda^{\infty}$-set and the projection image of a $\Lambda^{\infty}$-set are $\Lambda^{\infty}$-sets is constructive [14]. One can check that the following lemma holds true when one chase the proof of Speisseger's corresponding statements keeping Lemma 3.4 in mind. Hence, we omit the proof of it.

Lemma 3.5. Let $A, B, A^{\prime}$ and $B^{\prime}$ are $\Lambda_{\mathcal{D}}^{\infty}$-subsets of $\mathbb{R}^{n}$ such that $A$ and $A^{\prime}$ (resp. $B$ and $B^{\prime}$ ) are of the same form. Then the intersection (resp. union and difference) of $A$ and $B$ and the one of $A^{\prime}$ and $B^{\prime}$ are of the same form. In addition, the projection images of $A$ and $A^{\prime}$ are of the same form.

The following lemma is an immediate consequence of 5 of Lemma 3.4.

LEMma 3.6. Let $A$ be $a \Lambda_{\mathcal{D}}^{\infty}$-set and $k$ be a positive integer. Then there exists an integer $N$ such that the number of connected components of each fibre $\tilde{A}_{x}=\left\{y \in \mathbb{R}^{n-k}:(x, y) \in \tilde{A}\right\}$ is smaller than $N$ for any $x \in \mathbb{R}^{k}$ and for any $\Lambda_{\mathcal{D}}^{\infty}$-set $\tilde{A}$ which together with $A$ are of the same form. 
The following lemma follows from Lemmas 3.5 and 3.6. See [15], [16]. There, a simplex means a definable set which is definably homeomorphic to a simplex in the usual sense.

Lemma 3.7. Let $A$ be a compact $\Lambda_{\mathcal{D}}^{\infty}$-set. Let $B_{i}$ be locally closed $\Lambda_{\mathcal{D}}^{\infty}$ subsets of $A$ for $i=1, \ldots, k$. Then there exists a triangulation $T_{A}$ of $A$ compatible with $B_{i}$ for all $i$ whose simplicial complex is $\left\{\sigma_{1}^{A}, \ldots, \sigma_{k}^{A}\right\}$ (some of $\sigma_{j}^{A}$ 's may be empty, ) with the following property.

- Let $A^{\prime}$ be a compact $\Lambda_{\mathcal{D}}^{\infty}$-set such that $A$ and $A^{\prime}$ are of the same form. Let $B_{i}^{\prime}$ be locally closed $\Lambda_{\mathcal{D}}^{\infty}$-subsets of $A^{\prime}$, for $i=1, \ldots, k$, such that $B_{i}$ and $B_{i}^{\prime}$ are of the same form. Then there exists a triangulation $T_{A^{\prime}}$ of $A^{\prime}$ compatible with $B_{i}^{\prime}$ for all $i$ whose simplicial complex is $\left\{\sigma_{1}^{A^{\prime}}, \ldots, \sigma_{k}^{A^{\prime}}\right\}$ such that $\sigma_{j}^{A}$ and $\sigma_{j}^{A^{\prime}}$ are of the same form for all $j$.

Lemma 3.8. The quotient set of all definable subsets of an Euclidean space of the same form up to $\mathcal{P}(\tilde{\mathbb{R}})$-homeomorphism is finite.

Proof. Consider a $\mathcal{P}(\tilde{\mathbb{R}})$-definable set $S$. We may assume that $S$ is bounded. Set $S_{0}=\bar{S}, S_{1}=\overline{\bar{S} \backslash S}$ and $S_{i+1}=\overline{\overline{S_{i}} \backslash S_{i}}$ for $i \geq 1$. Then $S_{n+1}=\emptyset$ by o-minimality. Applying Lemma 3.7 to $S_{0}, S_{1}, \ldots, S_{n}$, we have shown that there exists a natural number $k$ such that any $\mathcal{P}(\tilde{\mathbb{R}})$-definable set which is of the form with $S$ is divided into $k \mathcal{P}(\tilde{\mathbb{R}})$-definable simplices.

Proof of Theorem 1.3. Two Pfaffian manifolds of Rolle type of the same family are of the same form by [14, Lemma 2.1]. Therefore, Theorem 1.3 is immediate from Lemma 3.8.

Proof of Theorem 1.4. A real analytic manifold is an analytic submanifold of some $\mathbb{R}^{n}$ by a Grauert's theorem. Especially, it is a subanalytic subset of $\mathbb{P}^{n}(\mathbb{R})$ under the canonical inclusion $\mathbb{R}^{n} \hookrightarrow \mathbb{P}^{n}(\mathbb{R})$ if it is compact. Therefore, Theorem 1.4 follows from Theorems 1.2 and 1.3.

\section{$\S 4$. Sketch of the proof of Theorem 1.5}

The proof of Theorem 1.5 is similar to that of Theorem 1.3. Hence, we also give a sketch of the proof of Theorem 1.5.

First, we begin in a general setting. Fix an o-minimal structure $\tilde{\mathbb{R}}$ on $\mathbb{R}$. We call a function $c$ from all definable sets to $\mathbb{N}$ complexity of $\tilde{\mathbb{R}}$. We also call the image $c(A)$ of a definable set $A$ under this function complexity of it. 
Definition 4.1. A complexity $c$ of the given o-minimal structure is calculable if the following conditions are satisfied.

c1 The complexity $c(A \cap B)$ of $A \cap B$ is bounded from above by the number determined only by the complexities $c(A)$ and $c(B)$ of definable sets $A, B \subset \mathbb{R}^{n}$.

c2 The complexity $c(A \cup B)$ is also less than the number determined by $c(A)$ and $c(B)$.

c3 The number $c\left(\mathbb{R}^{n} \backslash A\right)$ is smaller than the number determined by $c(A)$, where $A$ is a definable subset of $\mathbb{R}^{n}$.

c4 The number $c(A \times \mathbb{R})$ is smaller than the number determined by $c(A)$.

c5 Let $p: \mathbb{R}^{n} \rightarrow \mathbb{R}^{n-1}$ denote the projection forgetting the last factor. There exists a function from $\mathbb{N}$ to $\mathbb{N}$ such that $c\left(p^{-1}(x)\right)$ is smaller than the image of $n$ for all $x \in \mathbb{R}^{n-1}$.

c6 Let $p: \mathbb{R}^{n} \rightarrow \mathbb{R}^{n-1}$ be the same as above. Then $c(p(A))$ is bounded from above by the number determined only by $c(A)$.

c7 The number of connected components of a definable subset of $\mathbb{R}$ is smaller than the number determined only by the complexity of it.

We call a complexity of the graph of $f$ complexity of $f$ for short if $f$ is a definable function.

Example 4.2. Set $\overline{\mathbb{R}}:=\langle\mathbb{R},<, 0,1,+,-, \cdot\rangle$. Definable sets in $\overline{\mathbb{R}}$ are semi-algebraic sets. Any semi-algebraic set $S$ in $\mathbb{R}^{n}$ is of the form

$$
S=\bigcup_{i=1}^{k} \bigcap_{j=1}^{k}\left\{x \in \mathbb{R}^{n} ; f_{i j}(x) *_{i, j} 0\right\},
$$

where $f_{i j}$ are polynomials in $k$ indeterminates of total degree $\leq k$ and $*_{i j} \in\{<,=\}$. In this paper, the smallest $k$ satisfying the above conditions is a complexity of $\overline{\mathbb{R}}$ by definition. It is easy to check that it satisfies all the above axioms but c6. [4], [1] imply c6. Therefore, $\overline{\mathbb{R}}$ has a calculable complexity.

The known proofs of cylindrical definable cell decomposition and definable triangulation are constructive and one can easily check the following propositions using the above axioms. See [15], [16] for the proof of cylindrical definable cell decomposition and triangulation. 
Lemma 4.3. $\left(C^{s} \mathrm{CDCD}_{n}\right)$ Assume that $\tilde{\mathbb{R}}$ has a complexity c. A nonnegative integer $s$ is fixed.

Let $A_{1}, \ldots, A_{k}$ be definable subsets of $\mathbb{R}^{n}$. There exists a cylindrical definable $C^{s}$ cell decomposition compatible with $A_{1}, \ldots, A_{k}$ the number of whose cells is smaller than the number determined only by $c\left(A_{1}\right), \ldots, c\left(A_{k}\right)$, $n$ and $s$, and each complexity of whose cells $C$ and definable $C^{s}$ diffeomorphisms $\phi_{C}: C \rightarrow \mathbb{R}^{\operatorname{dim}(C)}$ is also bounded from above by the number determined by $c\left(A_{1}\right), \ldots, c\left(A_{k}\right), n$ and $s$.

LEMMA 4.4. Assume that $\tilde{\mathbb{R}}$ has a complexity c. Let $A$ be a compact definable set of $\mathbb{R}^{n}$ and $B_{i}$ be a locally closed definable subset of $A$ for each $i=1, \ldots, k$. Then there exists a definable triangulation of $A$ which is compatible with $B_{i}$ for all $i=1, \ldots, k$ and whose number of simplices is bounded from above by the number determined by $n, c(A)$ and $c\left(B_{i}\right)$, $i=1, \ldots, k$.

LEMMA 4.5. Assume that $\tilde{\mathbb{R}}$ has a complexity c. Given natural numbers $n$ and $d$. Then the number of topological types of all definable subsets $S$ of $\mathbb{R}^{n}$ with $c(S)=d$ is finite.

Proof. The proof of this lemma is the same as that of Lemma 3.8.

LEMMA 4.6. Let $M$ be a definable $C^{2}$ manifold and $\Omega=\left\{\omega_{1}, \ldots, \omega_{q}\right\}$ be definable $C^{1}$ differential 1-forms on $M$. Let $A$ be a definable subset of $M$. Then there exists a finite partition of $M$ into $C^{s}$ cells $(2 \leq s<\infty)$ compatible with $A$ such that, for each cell $C$ and $J \subset\{1, \ldots, q\}, \Omega_{J^{\prime}}$ is a basis of $\Omega_{J}$ along $C$ for some subset $J^{\prime} \subset J$. Furthermore, the number of cells of this partition is smaller than the number determined by $n$, complexities of $M, A$ and $\left\{a_{i, j}\right\}$, where $\omega_{i}=\sum_{j=1}^{n} a_{i, j} d x_{j}$. Each complexity of cells $C$ of this partition and the definable diffeomorphisms $\phi_{C}: C \rightarrow \mathbb{R}^{\operatorname{dim}(C)}$ are bounded from above by the number determined by complexities of them.

Proof. The first part of this lemma is [14, Lemma 2.8]. One can easily check the last part using Lemma 4.3 when one chase the proof of $[14$, Lemma 2.8].

In the rest of this section, we only consider the o-minimal structure $\overline{\mathbb{R}}$ of the real field. The calculable complexity of semialgebraic sets is given in Example 4.2. 
Set $\eta_{1}(\omega):=\max _{i=1, \ldots, n}\left(\right.$ complexity of $\left.a_{i}\right)$ for any semialgebraic $C^{1}$ differential 1-form $\omega=\sum_{i=1}^{n} a_{i}(x) d x_{i}$. Let $\Omega=\left\{\omega_{1}, \ldots, \omega_{q}\right\}$ be semialgebraic $C^{1}$ differential 1 -forms on $\mathbb{R}^{n}$ of full rank. Set secondly $\eta_{2}(V):=$ $\max \left\{q, \eta_{1}\left(\omega_{1}\right), \ldots, \eta_{1}\left(\omega_{q}\right)\right\}$ for any Pfaffian manifold $V$ of $\Omega$ of Rolle type. Set thirdly $\eta_{3}(W):=\max \left\{p,\left(\right.\right.$ complexity of $\left.A, \eta_{2}\left(V_{1}\right), \ldots, \eta_{2}\left(V_{p}\right)\right\}$ for any basic $\overline{\mathbb{R}}$-Pfaffian set $W=V_{1} \cap \cdots \cap V_{p} \cap A$. Set finally

$$
\eta(X):=\max \left\{s, \eta_{3}\left(W_{1}\right), \ldots, \eta_{3}\left(W_{s}\right)\right\}
$$

for any $\overline{\mathbb{R}}$-Pfaffian set $X=\bigcup_{i=1}^{s} W_{i}$, where $W_{1}, \ldots, W_{s}$ are basic $\overline{\mathbb{R}}$-Pfaffian sets.

Let $\Lambda_{\text {alg,n }}$ be the collection of all $\overline{\mathbb{R}}$-Pfaffian subset of $\mathbb{R}^{n}$. Set $\Lambda_{\text {alg }}=$ $\bigcup_{n \in \mathbb{N}} \Lambda_{\text {alg, } n}$. Then the family $\Lambda_{\text {alg }}$ satisfies the axioms of $\Lambda$-sets as pointed out in Section 2.

Lemma 4.7. 1. Let $X$ and $Y$ be $\overline{\mathbb{R}}$-Pfaffian sets of $\mathbb{R}^{n}$. Then $\eta(X \cap$ $Y)$ is less than the number determined only by $\eta(X)$ and $\eta(Y)$.

2. Let $X$ be an $\overline{\mathbb{R}}$-Pfaffian set of $\mathbb{R}^{n}$. Then $\eta(X \times \mathbb{R})$ is bounded from above by the number determined only by $\eta(X)$.

3. Let $X$ be the same as above and $\pi$ be a permutation of $\{1, \ldots, n\}$. Then $\eta(\pi(X))$ is also bounded from above by the number determined by $\alpha \in \eta(X)$.

4. Assume that non-negative integers $n$ and $m$ are given. There exist positive integers $n^{\prime}$ and $m^{\prime}$ with the following property.

- Let $X$ be an $\overline{\mathbb{R}}$-Pfaffian subset of $\mathbb{R}^{n}$ with $\eta(X) \leq m$. Then there exists a closed $\overline{\mathbb{R}}-$ Pfaffian set $Y$ with $\Pi(Y)=X$ and $\eta(Y) \leq m^{\prime}$, where $\Pi: \mathbb{R}^{n+n^{\prime}} \rightarrow \mathbb{R}^{n}$ is the projection forgetting the last $n^{\prime}$ factors.

5. Let $k$ and $m$ be non-negative integers. Then, for any $x \in \mathbb{R}^{k}$ and for any $\overline{\mathbb{R}}$-Pfaffian subset $X$ of $\mathbb{R}^{n}$ with $\eta(X) \leq m$, the number of connected components of $X_{x}=\left\{y \in \mathbb{R}^{n-k}:(x, y) \in X\right\}$ is smaller than the number determined only by $k$ and $m$.

6. Assume that non-negative integers $m$ and $n$ are given. Then, there exist positive integers $N$ and $m^{\prime}$ with the following property.

- Let $X$ be an $\overline{\mathbb{R}}$-Pfaffian subset of $\mathbb{R}^{n}$ with $\eta(X) \leq m$. Then there exist manifolds $B_{1}, \ldots, B_{k} \in \Lambda_{\text {alg,n }}$ in standard position with $k \leq N$ such that $X=\bigcup_{i=1}^{k} B_{i}$ and $\eta\left(B_{i}\right) \leq m^{\prime}$ for any $i=1, \ldots, k$. 
Proof. The statements 1, 2 and 3 are clear by the definition of $\eta$.

We show the statement 4 . We may assume that $X$ is a basic $\overline{\mathbb{R}}$-Pfaffian subset $A \cap V_{1} \cap \cdots \cap V_{p}$ of $\mathbb{R}^{n}$, where $A$ is a semialgebraic set. There exists a closed semialgebraic subset $B$ of $\mathbb{R}^{n+n^{\prime}}$ such that $\Pi(B)=A$. Furthermore, the number $n^{\prime}$ is determined by $n$ and $m$ and $\eta(B)$ is smaller than the number determined by $n$ and $m$. The set $Y=B \cap\left(V_{1} \times \mathbb{R}^{n^{\prime}}\right) \cap \cdots \cap\left(V_{p} \times \mathbb{R}^{n^{\prime}}\right)$ is a closed basic $\overline{\mathbb{R}}$-Pfaffian set. It is obvious that the relation $\Pi(Y)=X$ holds and $\eta(Y)$ is smaller than some number determined by $n$ and $m$.

The statement 5 follows from Corollary 2.4

We show the statement 6 last. We may assume that $X$ is a basic $\overline{\mathbb{R}}$ Pfaffian set $A \cap V_{1} \cap \cdots \cap V_{p} \subset \mathbb{R}^{n}$, where $A$ is a semialgebraic set and $V_{i}$ are Pfaffian manifolds of $\Omega_{i}$ of Rolle type. Apply Proposition 4.6 to $\bigcup_{i=1}^{p} \Omega_{i} \cup\left\{d x_{1}, \ldots, d x_{n}\right\}$. We obtain a partition $\mathcal{P}$. Then the manifold $W_{C}:=C \cap V_{1} \cap \cdots \cap V_{p}$ is in standard position for any cell $C$ of $\mathcal{P}$ by the proof of [14, Corollary 2.9]. Furthermore, the number of cells is smaller than the number determined by $n$ and $m$, and $\eta(C)$ is bounded from above by the number determined by $n$ and $m$ for any cell $C$.

A $\Lambda_{\text {alg }}^{\infty}$-set is a $\Lambda^{\infty}$-set obtained from $\overline{\mathbb{R}}$-Pfaffian sets. Let $Y$ be a $\Lambda_{\mathrm{alg}}^{\infty}$-set with $Y:=\bigcup_{i=1}^{r} X_{i}$, where $X_{i}$ are basic $\Lambda_{\text {alg }}^{\infty}$-sets obtained from $\overline{\mathbb{R}}$-Pfaffian sets $W_{i}$. We define the number $\kappa(Y)$ as follows.

$$
\kappa(Y):=\max \left\{r, \eta\left(W_{1}\right), \ldots, \eta\left(W_{r}\right)\right\}
$$

LEMma 4.8. The function $\kappa$ defined above is a complexity of the ominimal structure constructed from all Pfaffian manifolds of Rolle type over $\overline{\mathbb{R}}$.

Proof. We have only to check that $\kappa$ satisfies all the axioms of a complexity. They are proved by repeating the proof of [14] using Lemma 4.7 in the same way as Lemma 3.5 and Lemma 3.6. We omit the proof.

Proof of Theorem 1.5. The first part is an immediate consequence of Corollary 1.1 and Theorem 1.2. The last part follows from Theorem 4.5 and Lemma 4.8.

\section{§5. Pfaffian manifolds of non-Rolle type}

The purpose of this section is to prove Proposition 1.6 and to construct manifolds and differential 1-forms which satisfy the conditions of Theorem 1.9 and Theorem 1.10.

We show Proposition 1.6 first. 
Proof of Proposition 1.6. Let $\mathcal{T}$ be a triangulation of $\bar{M}$ compatible with $M$. Let $\sigma$ be a definable open simplex contained in $M$ with $\operatorname{dim} \sigma<$ $\operatorname{dim} M$. The set

$$
U_{\sigma}=\sigma \cup \bigcup \tau
$$

where the large union is taken over all open simplices of $\mathcal{T}$ with $\operatorname{dim}(\tau)>$ $\operatorname{dim}(\sigma)$ and $\sigma \subset \bar{\tau}$, is a simply connected open set containing $\sigma$. Then open covering of $M$ consisting of all open simplices of $\mathcal{T}$ of $\operatorname{dimension}=\operatorname{dim} M$ and all open sets of the form $U_{\sigma}$ satisfies the conditions of the proposition by Haefliger $([5])$.

Proposition 1.7 and 1.8 are direct corollaries of Proposition 1.6.

We show Theorem 1.9 next.

Proof of Theorem 1.9. Let $M_{1}$ be a Nash manifold which is Nash diffeomorphic to a 2-dimensional open disc. Let $M_{2}$ be a Nash manifold which is Nash diffeomorphic to $S^{1} \times \mathbb{R}$. We construct a Nash manifold $M_{3}$ by sticking two strips on a Nash manifold which is Nash diffeomorphic to $S^{1} \times \mathbb{R}$ in the following way. Set $V_{1}=\left\{(x, y) \in \mathbb{R}^{2}: 1 / 4<x^{2}+y^{2}<1\right.$ or $(-1 / 4<$ $x<1 / 4,-1 / 2<y<1 / 2)\}, V_{2}=(-1 / 2,1 / 2) \times(-1 / 4,1 / 4)$ and $V^{\prime}=$ $\left\{1 / 4<x^{2}+y^{2}<1\right\} \cap(((-1 / 2,-1 / 4) \cup(1 / 4,1 / 2)) \times(-1 / 4,1 / 4)) \subset V_{1}$. We define the manifold $M_{3}$ by sticking open sets $V_{2}$ on $V_{1}$ by the mapping $\varphi: V^{\prime} \rightarrow V_{2}$ given by $\varphi(x, y, z):=(x, y, z)$.

Set $A:=M_{1} \times(0, \infty), B:=M_{2} \times(0, \infty)$ and $C:=M_{3} \times(0, \infty)$. The sets $A, B$ and $C$ can be regarded as 'towers' with $(0, \infty)$ 'floors' such that each 'floor' of $A, B$, and $C$ is $M_{1}, M_{2}$ and $M_{3}$, respectively. Let $M_{4}$ be a Nash manifold which is Nash diffeomorphic to a strip $\mathbb{R}^{2}$. Paste six 'passages' $M_{4} \times(0, \infty)$ and 'towers' in the following way.

The Passage1 links $(1 / 9,1 / 2)$ 'floors' of $A$ and $(2 / 9,1)$ 'floors' of $A$.

The Passage 2 links $(1 / 9,1 / 3)$ 'floors' of $A$ and $(1 / 3,1)$ 'floors' of $A$.

The Passage3 links $(1 / 2,1)$ 'floors' of $A$ and $(3 / 2,3)$ 'floors' of $B$.

The Passage4 links $(1 / 3,1 / 2)$ 'floors' of $A$ and $(1,3 / 2)$ 'floors' of $C$.

The Passage5 links $(3 / 2, \infty)$ 'floors' of $B$ and $(3 / 2, \infty)$ 'floors' of $B$.

The Passage6 links $(1,3 / 2) \cup(3 / 2, \infty)$ 'floors' of $C$ and $(3,9 / 2) \cup$ $(9 / 2, \infty)$ 'floors' of $C$.

If we proceed from the $p$-th 'floor' along the Passage1, we reach the $2 p$-th 'floor'. We reach the $3 p$-th 'floor' if we proceed from the $p$-th 'floor' along the other 'passages'. 
Figure 1 represents the $p$-th floors of Tower $A, B, C$ and the connections among them by 'passages'. Let $X$ be the Nash manifold constructed above which has three 'towers' and six 'passages'.
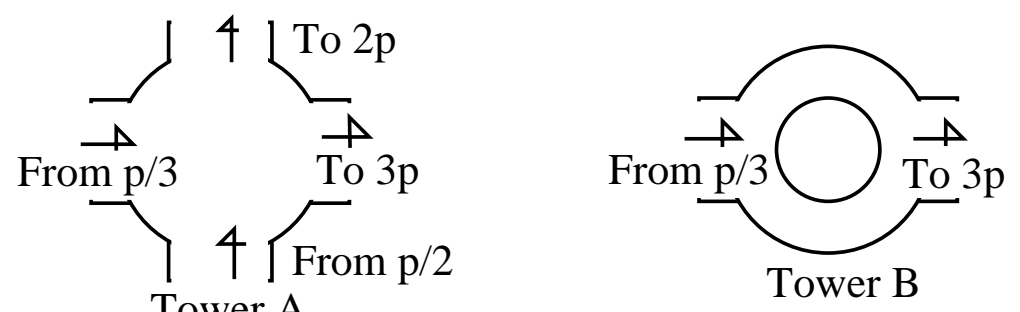

Tower A

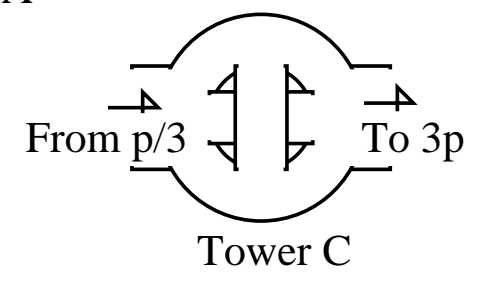

Figure 1: The 'floor' of each 'towers'.

Let $\omega_{A}, \omega_{B}$ and $\omega_{C}$ be a canonical analytic differential 1-form on $A$, $B$ and $C$ whose Pfaffian manifolds are 'floors', respectively. There exists an integrable semialgebraic $C^{2}$ differential 1-form $\omega$ on $X$ which equals $\omega_{A}$, $\omega_{B}$ and $\omega_{C}$ on $A, B$ and $C$, respectively, and whose Pfaffian manifold $X_{p}$ containing the $p$-th 'floor' is just the reachable region from the $p$-th 'floor' passing 'passages'.

Figure 2 represents the shape of $X_{\frac{13}{72}}$. Let $m$ be an integer. When one goes up the Passage1 just $m$-times and Passage2 some times from the $p$-th floor of Tower A and goes up the Passage3 or Passage4 last, one reaches Tower B or Tower C. If $m$ is negative, we go down the Passage1 just $-m$ times. For example, we can reach Tower B if we go up Passage1 just twice from the 13/72-th floor. In this way, for any $p$, we can construct a mapping from $\mathbb{Z}$ onto $\{B, C\}$. This mapping is expected to be a topological invariant. We will prove it.

We define the equivalence relation $\simeq$ on positive real numbers. The relation $p \simeq p^{\prime}$ holds by definition if there exist two integers $m$ and $n$ with $p^{\prime} / p=2^{m} 3^{n}$. Note that, if $p \simeq p^{\prime}$ and $p \nsucceq 1$, then $X_{p}=X_{p^{\prime}}$. We claim that there exists at most one another leaf of the foliation defined by $\omega$ which is homeomorphic to $X_{p}$ if $p \not 1$. The manifold $X$ and the differential 1form $\omega$ are the required ones, if the claim is proved, because $X$ is an affine 


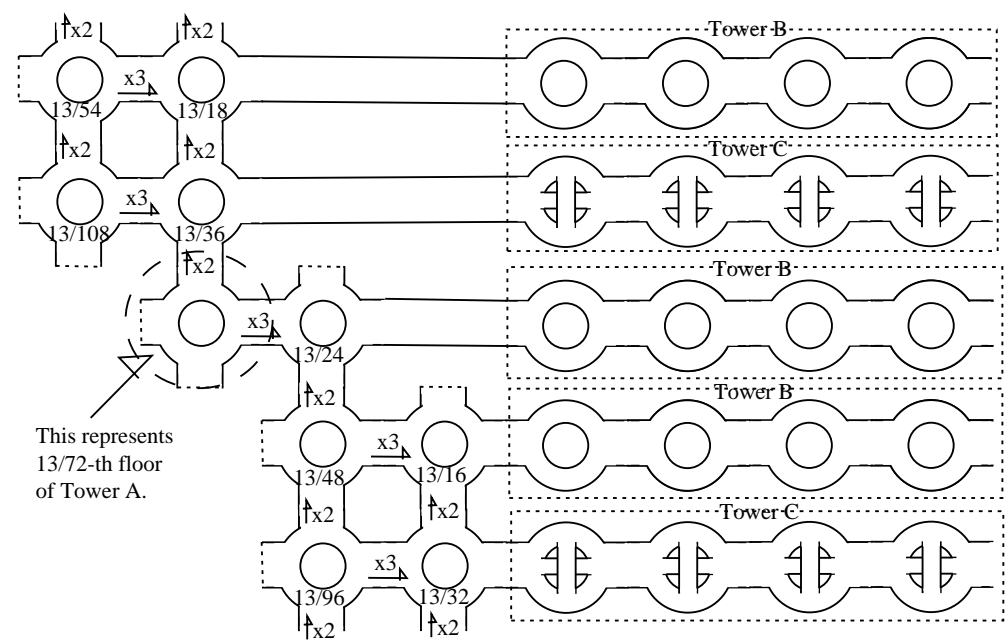

Figure 2: The shape of $X_{\frac{13}{72}}$.

semialgebraic $C^{2}$ manifold by [12, Theorem III.1.1].

Fix a Pfaffian manifold $X_{p}$ with $p \not 1$.

Set $\alpha:=\left\{(x, y) \in \mathbb{R}^{2}: 1<x^{2}+y^{2} \leq 4\right\}$. Two proper embeddings $f_{1}^{\prime}, f_{2}^{\prime}: \alpha \rightarrow X_{p}$ are equivalent if there exist proper embeddings $f_{3}^{\prime}: \alpha \rightarrow X_{p}$ and $g_{1}^{\prime}, g_{2}^{\prime}: \alpha \rightarrow \alpha$ with $f_{3}^{\prime}=f_{1}^{\prime} \circ g_{1}^{\prime}$ and $f_{3}^{\prime}=f_{2}^{\prime} \circ g_{2}^{\prime}$. This relation is an equivalence relation. Let $S_{\alpha}$ be the set of all equivalence classes. We choose one representative for each element of $S_{\alpha}$. Then we can construct a new manifold $D X_{p}$ by sticking $D:=\left\{(x, y) \in \mathbb{R}^{2}: x^{2}+y^{2} \leq 4\right\}$ on $X_{p}$ by the chosen proper embeddings. Another choice of representatives makes another manifold. We don't care about the choice of representatives because we only need the common property of $D X_{p}$ which is not effected by the choice.

Set $\beta:=\left\{(x, y) \in \mathbb{R}^{2}: 0<y \leq 1\right\}$. Let $f_{1}, f_{2}: \beta \rightarrow X_{p}$ be proper embeddings such that the composition of the inclusion $X_{p} \hookrightarrow D X_{p}$ and $f_{1}$ (resp. the one of the inclusion and $f_{2}$ ) is also a proper embedding. Two proper embeddings $f_{1}, f_{2}: \beta \rightarrow X_{p}$ are equivalent if there exist proper embeddings $f_{3}: \beta \rightarrow X_{p}$ and $g_{1}, g_{2}: \beta \rightarrow \beta$ with $f_{1}=f_{3} \circ g_{1}$ and with $f_{2}=f_{3} \circ g_{2}$. This relation is an equivalence relation by the construction of the manifold $D X_{p}$. The notation $[f]$ denotes the equivalence class of $f$. Set $S_{\beta}:=\{[f]\}$. Two proper embeddings $f_{1}, f_{2}: \beta \rightarrow X_{p}$ are equivalent including boundary if there exist proper embeddings $f_{3}: \beta \rightarrow X_{p}$ and $g_{1}, g_{2}$ : $(\bar{\beta}, \beta) \rightarrow(\bar{\beta}, \beta)$ with $f_{1}=f_{3} \circ g_{1}$ and with $f_{2}=f_{3} \circ g_{2}$. The notation $[[f]]$ 
denotes the equivalence class of $f$ under the equivalence relation including boundary. Set $S_{\beta}^{r}:=\{[[f]]\}$. Then there exists a natural map $\varpi: S_{\beta}^{r} \rightarrow S_{\beta}$. Let $T$ be a subset of $S_{\beta}^{r}$ such that the map $\left.\varpi\right|_{T}: T \rightarrow S_{\beta}$ is a bijection. We can construct a manifold $(B D X)_{p}$ with boundary by pasting $D X_{p}$ and $\bar{\beta}$ by the elements of $T$. The notation $[[f]]$ also denotes the boundary determined by $[[f]]$ later. Figure 3 represents $(B D X)_{\frac{13}{72}}$. The exceptional boundary and $[[f]]$ are also figured in Figure 3.

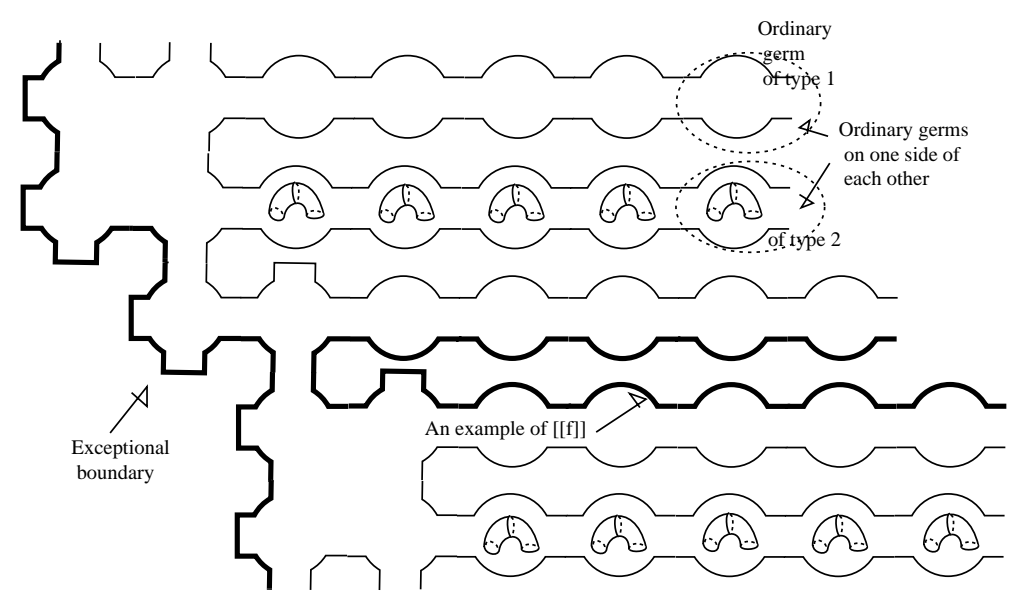

Figure 3: $(B D X)_{\frac{13}{72}}$.

Let $f_{1}, f_{2}: \beta \rightarrow(B D X)_{p}$ be proper embeddings. We say that $f_{1}$ and $f_{2}$ are equivalent if the last equivalence condition is satisfied replacing $X_{p}$ with $(B D X)_{p}$. We can construct a manifold $(C D X)_{p}$ in the same way as $(B D X)_{p}$. Let $T$ be the set defined in the construction of $(B D X)_{p}$. By the construction, there exist natural inclusions $X_{p} \hookrightarrow(B D X)_{p} \hookrightarrow(C D X)_{p}$. Only one connected component of $(C D X)_{p} \backslash X_{p}$ equals some connected component $E_{p}$ of $(B D X)_{p} \backslash X_{p}$. There exists only one element $[[\xi]] \in T$ such that $\xi(\beta) \cup E_{p}$ is connected for any representative $\xi$ of $[[\xi]]$. We call such $[[\xi]]$ an exceptional boundary.

The above definitions are the substitutions of the boundary in the natural sense. The first equivalence relation corresponds to the boundary homeomorphic to a circle $S^{1}$ and the others are correspondences of the one homeomorphic to $\mathbb{R}$.

We define the germ of $D X_{p}$ at infinity. Let $F$ and $G$ be connected subsets of $D X_{p}$. These are equivalent if there exists a compact set $K$ of 
$(B D X)_{p}$ such that $F \backslash K=G \backslash K$ and the sets $F \backslash K^{\prime}$ and $G \backslash K^{\prime}$ are connected for any compact subset $K^{\prime}$ of $(B D X)_{p}$ containing $K$. An equivalence class under this equivalent relation is called a germ at infinity. We define the relation of inclusion. Let $\zeta_{1}$ and $\zeta_{2}$ be germs at infinity. A germ $\zeta_{1}$ at infinity is contained in $\zeta_{2}$ if, for any representative $F_{1}$ of $\zeta_{1}$, there exists a representative $F_{2}$ of $\zeta_{2}$ with $F_{1} \subset F_{2}$. A maximal germ at infinity is a germ at infinity which is maximal with respect to this inclusion relation.

A maximal germ $\zeta_{1}$ at infinity is on one side of $\zeta_{2}$ if there exists a proper curve $\gamma:[0, \infty) \rightarrow X_{p}$ with the following properties.

- $F \cap \gamma([0, \infty)) \neq \emptyset$ and $G \cap \gamma([0, \infty)) \neq \emptyset$ for any representatives $F$ and $G$ of $\zeta_{1}$ and $\zeta_{2}$, respectively.

- Let $\zeta$ be a maximal germ at infinity distinct from $\zeta_{1}$ and $\zeta_{2}$. Then $H \cap \gamma([0, \infty))=\emptyset$ for some representative $H$ of $\zeta$.

- There exists only one element $[f]$ of $S_{\beta}$ such that $f(\beta) \cap \gamma([0, \infty))$ is not empty for any representative $f: \beta \rightarrow X_{p}$ of $[f]$.

A maximal germ $\zeta$ at infinity is an ordinary germ at infinity if $F \cup[[\xi]]$ is not connected for some representative $F$ of $\zeta$, where $[[\xi]]$ is the exceptional boundary. Remark that there exist two ordinary germs at infinity which are on one side of a given ordinary germ at infinity.

An ordinary germ at infinity $\zeta$ is of type 1 if the homotopy group $\pi_{1}(F)$ is a group consisting of a single element for some representative $F$ of $\zeta$. An ordinary germ at infinity $\zeta$ is of type 2 if it is not of type 1 . The germ $\xi$ at infinity of type 1 and the one of type 2 never map onto each other by any homeomorphism from $D X_{p}$ to $D X_{p}$ because homotopy is a topological invariant.

We construct a new topological invariant.

We define first the equivalence relation on all maps from $\mathbb{Z}$ to $\{1,2\}$. Let $\tilde{\kappa}$ and $\tilde{\kappa}^{\prime}$ be maps from $\mathbb{Z}$ into $\{1,2\}$. Maps $\tilde{\kappa}$ and $\tilde{\kappa}^{\prime}$ are equivalent if there exist integers $l \in \mathbb{Z}$ and $m \in\{-1,1\}$ with $\tilde{\kappa}(i)=\tilde{\kappa}^{\prime}(m i+l)$ for any $i \in \mathbb{Z}$.

Let $\kappa: \mathbb{Z} \rightarrow\{$ ordinary germ at infinity $\}$ be a map such that $\kappa(i)$ and $\kappa(i+1)$ are on one side of the other for any $i \in \mathbb{Z}$. Define the map $\tilde{\kappa}: \mathbb{Z} \rightarrow$ $\{1,2\}$ as follows.

$$
\tilde{\kappa}(i)= \begin{cases}1 & \text { if } \kappa(i) \text { is of type } 1 \\ 2 & \text { if } \kappa(i) \text { is of type } 2 .\end{cases}
$$

It is obvious that the equivalence class of $\tilde{\kappa}$ is the topological invariant. The equation $\tilde{\kappa}(i)=1$ holds if and only if we reach the 'tower' $B$ proceeding 
from some fixed 'floor' in $A$ along the Passage1 just $i$ times.

We calculate this invariant for $X_{p}$ with $p \not 11$. Let $\eta(i)$ be the largest integer which does not exceed the number $-\frac{\log p}{\log 2}-\frac{\log 3}{\log 2} i$ for any $i \in \mathbb{Z}$. Then

$$
\tilde{\kappa}(i)= \begin{cases}1 & \text { if } 1 / 2<2^{\eta(i)} 3^{i} p<1 \\ 2 & \text { if } 1 / 3<2^{\eta(i)} 3^{i} p<1 / 2 .\end{cases}
$$

Therefore, there exists at most one $p^{\prime}$ such that $X_{p^{\prime}}$ is homeomorphic to $X_{p}$.

We show Theorem 1.10 last.

Proof of Theorem 1.10. We construct a compact real analytic manifold and an analytic differential 1-form on it such that there exist Pfaffian manifolds of it which are homeomorphic to $X_{p}$ for all $p$ with $1 / 9<p<1$. Let $M$ be a compact Nash manifold of dimension 2 given in Figure 4 . Let

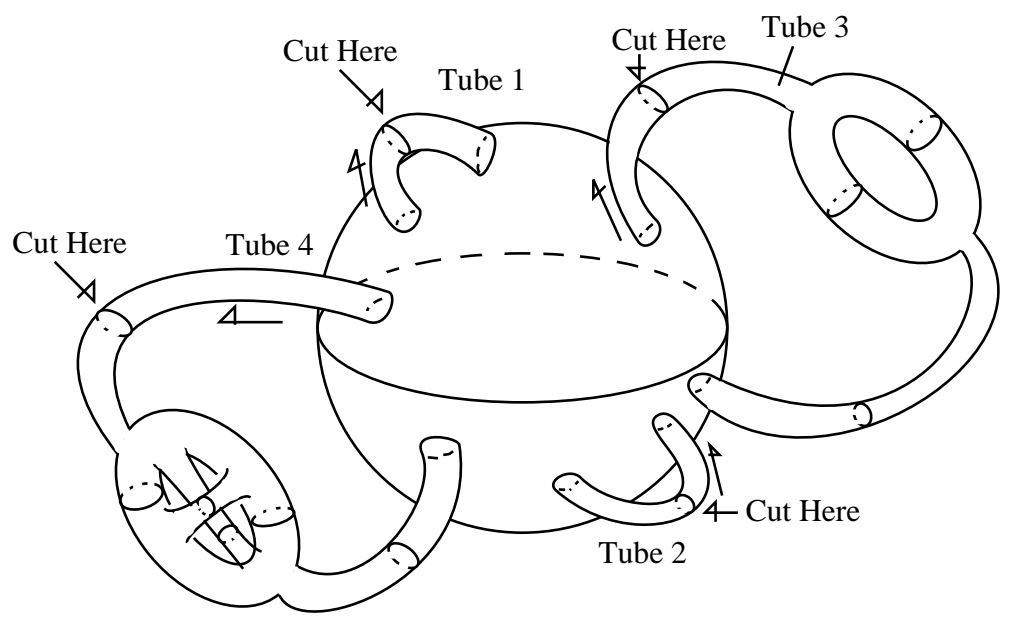

Figure 4: The shape of $M$.

$\psi: M \rightarrow \mathbb{R}$ be an analytic function such that a connected component of $\psi^{-1}(\{x \in \mathbb{R}: x \neq 0\})$ is analytically diffeomorphic to the analytic manifold in Figure 5. It can be chosen by the Weierstrass-Stone theorem. Let $\left\{\left(U_{i}, \varphi_{i}\right)\right\}_{i=1,2}$ be an atlas of $S^{1}$ defined as follows.

$$
\begin{gathered}
\varphi_{2} \circ \varphi_{1}: \varphi_{1}^{-1}\left(U_{1} \cap U_{2}\right)=\mathbb{R} \backslash\{0\} \longrightarrow \mathbb{R} \backslash\{0\}=\varphi_{2}^{-1}\left(U_{1} \cap U_{2}\right), \\
\varphi_{2} \circ \varphi_{1}(x)=1 / x .
\end{gathered}
$$




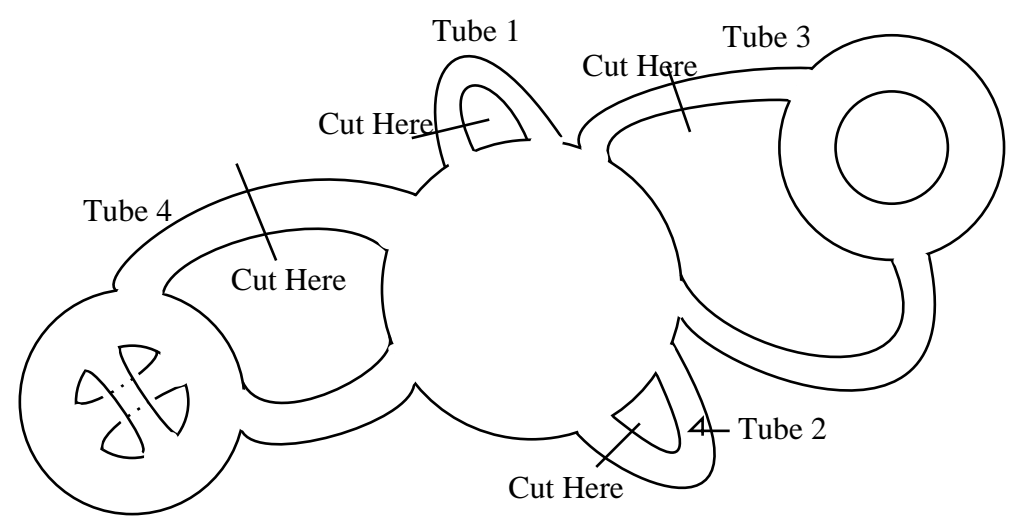

Figure 5: A connected component of $(\psi)^{-1}(\{x \in \mathbb{R} ; x \neq 0\})$.

For any $r \in \mathbb{R}$ with $r \neq 0$, let $t_{r}: S^{1} \rightarrow S^{1}$ be an analytic mapping defined by $\varphi_{1}^{-1} \circ t_{r} \circ \varphi_{1}(x)=r x$. Let $\Pi: M \times S^{1} \rightarrow S^{1}$ be the natural projection. Let $\omega$ be an analytic differential 1-form on $M \times S^{1}$ whose Pfaffian manifolds are of the form $\Pi^{-1}(x)$.

Cut tube 1 , tube 2 , tube 3 and tube 4 at the 'Cut Here' points in Figure 4 and Figure 5. Join tube 1 in $\Pi^{-1}(x)$ with tube 1 in $\Pi^{-1}\left(t_{2}(x)\right)$. Join tube 2 (resp. tube 3 , tube 4 ) in $\Pi^{-1}(x)$ with tube 2 (resp. tube 3 , tube 4$)$ in $\Pi^{-1}\left(t_{3}(x)\right)$. The constructed manifold $\tilde{M}$ is a compact Nash manifold and the function $\psi$ defined above can be regarded as an analytic function on $\tilde{M}$ naturally.

Let $g$ be an analytic Riemann metric on $M$. Identify $\omega$ with its dual. Then the differential 1-form $\omega / g(\omega, \omega)$ on $\tilde{M}$ is well-defined and analytic. Set $\tilde{\omega}=\omega / g(\omega, \omega)$. We identify the subset $U_{1}$ of $S^{1}$ with $\mathbb{R}$. We may assume that the manifold $\tilde{M}$ is a 'tower' with four 'passages' as in the case of Theorem 1.9. Four 'passages' are tubes. The $\infty$-th 'floor' is by definition the fibre $\Pi^{-1}\left(S^{1} \backslash U_{1}\right)$.

Consider an analytic mapping from $\tilde{M}$ to $S^{1}$ which sends an arbitrary point to its 'floor' number. From it, we can construct an analytic function $\rho: \tilde{M} \rightarrow \mathbb{R}$ whose zero set $L$ satisfies the following conditions.

Let $T_{p}^{1}$ be the tube 1 joining the $p$-th 'floor' and the $2 p$-th 'floor'. Let $T_{p}^{2}, T_{p}^{3}$ and $T_{p}^{4}$ be the tube 2,3 and 4 joining the $p$-th 'floor' and the $3 p$-th 'floor', respectively. Pairs $\left(T_{p}^{i} \cap L, T_{p}^{i}\right)$ are analytically diffeomorphic to one of $\left(\emptyset, S^{1} \times \mathbb{R}\right),\left(S^{1} \times\{0\}, S^{1} \times \mathbb{R}\right)$ and $\left(S^{1} \times\{-1,1\}, S^{1} \times \mathbb{R}\right)$. The number of connected components of $L \cap T_{p}^{i}$ is 
- 0 if $p<1 / 9,1$ if $p=1 / 9, \infty$ and 2 if $p>1 / 9$ in case where $i=1$,

- 2 if $p=\infty, p<1 / 9$ or $1 / 3<p, 1$ if $p=1 / 9,1 / 3$ and 0 if $1 / 9<p<1 / 3$ in case where $i=2$,

- 0 if $p>1,1$ if $p=1, \infty$ and 2 if $p<1$ in case where $i=3$ and

- 0 if $p>3 / 2,1$ if $p=3 / 2, \infty$ and 2 if $p<3 / 2$ in case where $i=4$.

We may assume that the manifold $\tilde{M}$ is an affine analytic manifold and the differential 1-form $\psi \rho \tilde{\omega}$ is analytic on $\tilde{M}$ by Grauert's theorem. Therefore, $\tilde{M}$ and $\psi \rho \tilde{\omega}$ are the required ones.

\section{REFERENCES}

[1] S. Basu, R. Pollack and M.-F. Roy, On the combinatorial and algebraic complexity of quantifier elimination, Journal of ACM, 43 (1996), no. 6, 1002-1045.

[2] F. Chazal, Sur les feuilletages algébriques de Rolle, Comment. Math. Helv., 72 (1997), 411-425.

[3] F. Chazal, Strcture locale et globale des feuilletage de Rolle, un théorème de fibration, Ann. Inst. Fourier, 48 (1998), no. 2, 553-592.

[4] G. E. Collins, Quantifier elimination for real closed fields by cylinderical algebraic decomposition, Springer Lecture Notes in Computer Science 33, pp. 515-532.

[5] A. Haefliger, Structures feuilletées et cohomologie á valeurs dans un faisceau de groupöide, Comment. Math. Helv., 32 (1958), 248-329.

[6] A. Khovanskii, On a class of systems of trancendental equations, Soviet Math. Dokl., 22 (1980), no. 3, 762-765.

[7] J.-M. Lion, Étude des hypersurfaces Pfaffiennes de Rolle, PhD thesis, Université de Bourgogne (1991).

[8] J.-M. Lion and J.-P. Rolin, Volumes, feuilles de Rolle et feuilletages analytiques réeles et théorème de Wilkie, Ann. Toulouse, 7 (1998), 93-112.

[9] J.-M. Lion and P. Speissegger, Analytic strafication in the pfaffian closure of an o-minimal structure, Duke Math. J., 103 (2000), no. 2, 215-231.

[10] R. Moussu and C. Roche, Théorie de Khovanskii et problème de Dulac, Invent. Math., 105 (1991), 431-441.

[11] R. Moussu and C. Roche, Théorème de finitude uniformes pour les variétés pfaffiennes de Rolle, Ann. Inst. Fourier, 42 (1992), 393-420.

[12] M. Shiota, Nash manifolds, Lecture Notes in Mathematics 1269, Springer-Verlag, 1980.

[13] M. Shiota, Geometry of subanalytic and semialgebraic sets, Birkhäuser, 1997.

[14] P. Speissegger, The Pfaffian closure of an o-minimal structure, J. reine angew. Math., 508 (1999), 189-221.

[15] L. van den Dries, Tame topology and O-minimal structure, London Math. Soc. Lecture Note 248, Cambridge Univ. Press, 1998.

[16] L. van den Dries and C. Miller, Geometric categories and o-minimal structures, Duke Math. J., 84 (1996), 497-540. 
[17] A. J. Wilkie, Model completeness results for expansions of the ordered field of real numbers by restricted pfaffian functions and the exponential function, J. Amer. Math. Soc., 9 (1996), 1051-1094.

Masato Fujita

Department of Mathematics

Graduate School of Science

Kyoto University

Sakyou Kyoto, 606-8502

Japan

fujita@kusm.kyoto-u.ac.jp

Masahiro Shiota

Graduate School of Mathematics

Nagoya University

Chikusa Nagoya, 464-8601

Japan

shiota@math.nagoya-u.ac.jp 